\title{
Die Bedeutung der Urkunde zur Weihe der St. Marien-Kirche in Zwickau 1118 für die Erforschung der Besiedlung des Erzgebirges
}

\author{
Karlheinz Hengst
}

1 Vgl. Walter Schlesinger: Kirchengeschichte Sachsens im Mittelalter. Von den Anfängen kirchlicher Verkündigung bis zum Ende des Investiturstreits. Köln, Graz 1962, Bd. I, S. $185 \mathrm{f}$.

2 Vgl. ausführliche Darstellung bei Karl Streller: Die Besiedlung und die territoriale Entwicklung des ehemaligen Gaues Zwickau von den Anfängen bis zum Ausgang des Mittelalters, in: Herbergen der Christenheit 1971, S. 42-125.

3 Felix Rosenfeld: Urkundenbuch des Hochstifts Naumburg. Teil I. Magdeburg 1925, Nr. 116, S. 101 f.; Codex diplomaticus Saxoniae regiae Reihe I, Bd. 2, Nr. 53, S. 45.

4 Die Lesart „Recma“ in den Urkundeneditionen ist wohl durch „Recina" zu ersetzen. Dies kann eine Wiedergabe des slawischen „Rěčina“ im Sinne von ,gewässerreiche Gegend sein. Wahrscheinlicher ist aber wohl als Ausgangsform das slawische „Rěčsna“ [gora] wörtlich ,Flussberg“, also ein Verweis auf einen Berg an einem Gewässer.
2018 begeht Zwickau das 900-jährige Jubiläum seiner ersten Marienkirche. Sie befand sich damals in dem im späteren Stadtbild von Zwickau aufgegangenen Osterwe(i)n. ${ }^{1}$ Heute erinnert an diese slawische Siedlung nur noch die Osterweihstraße in der Nähe der Moritzkirche. Der heutige Dom St. Marien ist somit Nachfolger jener ältesten Kirche an neuem Standort. ${ }^{2}$ Über die 1118 vom Bischof von Naumburg vollzogene Weihe ist glücklicherweise eine Urkunde erhalten geblieben. Es handelt sich dabei um eine zuverlässige $\mathrm{Ab}$ schrift aus dem Jahr 1598 vom damals noch vorhandenen Original, das als verloren gilt. ${ }^{3}$ Inhalt der von Bischof Dietrich von Naumburg (gest.1123) gezeichneten Urkunde ist, dass er auf Veranlassung und Wunsch der Gräfin Bertha von Groitzsch in ihrem „territorium Zwikowe" eine Parochialkirche zu Ehren der Heiligen Jungfrau Maria geweiht, und sie in den Verantwortungsbereich des Klosters Bosau bei Zeitz überwiesen hat. In der Urkunde wird sogleich ausdrücklich auf die von Anfang an geplante Zuständigkeit der neuen Kirche nicht für einen Ort, sondern ein größeres Gebiet verwiesen. Zur materiellen Ausstattung der Kirche enthält die Urkunde insbesondere die Nennung von zwei Hufen sowie den Böhmischen Zoll als jährliche Einnahme - und sechs geistliche Brüder sollen in der Kirche ihren Dienst tun.

\section{Die Grenzbeschreibung der Parochie 1118 und die ältesten Namen}

Nach diesen generellen Angaben wird das Territorium der künftigen Parochie in seiner Ausdehnung gekennzeichnet. Demnach bildete der Mülsenbach („rivulus Milsena“) von der Quelle bis zur Mündung bei Schlunzig in die Mulde (,in Muldam“) die Ostgrenze. Im Süden wird die Grenzlinie von einem Berg mit heute unbekanntem Namen („mons Luderni“) hin zur Mündung „Scurnice in Muldam collemque Recina“ gezogen. Die Form „Scurnice“ zeigt den Genitiv an zu „Scurnica“, was die slawische Form für ein ,schwarzes Gewässer' (heutiges Schwarzwasser) ist, wiederum mit Mündung in die Mulde. Der Berg „Recina“4 mit ebenfalls slawischem Namen weist auf seine Lage an einem Bach oder Fluss oder eventuell auf einen an Fließgewässern reichen Berg hin, dann also wohl einen Berg mit Quellen für mehrere Wasserläufe.

Es ist anzunehmen, dass die Grenzbeschreibung zum Süden von Ost nach West erfolgte. Folglich ist der Berg „mons Luderni“ zunächst näher zu bestimmen. Über den Namen ist schon viel gerätselt worden. In der lateinisch geschriebenen Urkunde mutet der Name an, als handle es sich um den Namen eines Besitzers. Doch dem ist nicht so. Vielmehr liegt hier eine aus dem deutschen Sprachgebrauch übernommene Form zugrunde. Die mittelhochdeutschen Wörter „lûder, luoder“ ,Tierkadaver, Aas“ sowie mittelhochdeutsch „luodern“ ,locken` bieten die Klärung des rätselhaften Bergnamens. Im Mittelalter war es bereits üblich, dass Jäger ein totes Tier zum Anlocken von Raubtieren auslegten. In der Jägersprache ist der „Luderplatz“ der Fachausdruck für eine solche Stelle im Gelände. Bei dem „mons Luderni“ geht es folglich um einen ,Berg mit einer Stelle für ausgelegte Tierkadaver'. Geprägt haben diesen Namen sehr wahrscheinlich die im Raum von Mulde und Chemnitz-Fluss seit dem 11. Jahrhundert für die Wegesicherung nach Böhmen hin zuständigen Wolfsjäger („venatores luporum“). Zu suchen sein könnte dieser Berg südlich von der Quelle des Mülsenbachs, etwa die „Hohe Warte“ (502 m) zwischen Mulde und Alberoda westlich Lößnitz. Damit ließe sich die Grenzbeschreibung nach dem Urkundentext dann sinnvoll fortführen zur Mündung des Schwarzwassers in die Mulde (bei Aue) und von da weiter nach Westen 
zu dem schwer bestimmbaren Berg „Recina“. Vielleicht ist es der heutige Krähenberg (441 m) am Rödelbach in Saupersdorf oder eventuell der Borberg (433 m), der aber doch ein ganzes Stück entfernt vom Rödelbach liegt. Zu beachten ist, dass die Grenzbeschreibung mit Ebersbrunn bei Zwickau fortgesetzt wird, der Berg folglich am ehesten in der Nähe von Kirchberg zu wähnen ist.

Die Westgrenze wird in ihrem Verlauf von Süd nach Nord angegeben mit einer Quelle „Albodistudinza dicitur“ (Albolds Brunnen/Quelle) genannt, wohl nach einem dort ansässig gewordenen Albold) ${ }^{5}$, heutiges Ebersbrunn, wobei sicher die Quelle westlich vom heutigen Ort mit einem Bachlauf durch Schönfels und Gospersgrün gemeint ist. Und diesem Gewässer folgend reicht die Westgrenze bis hin zur Mündung in die Pleiße (,decensum [...] in Plisnam“) bei Steinpleis. Die Kürze dieser Westgrenze ist auffällig. Sie schwenkte dort sehr wahrscheinlich zur Mulde, vermutlich etwa bei Crossen, und folgte dann offenbar der Mulde flussabwärts bis zur Mündung des Mülsenbaches in die Mulde bei Schlunzig. Damit ist eigentlich das Terrain umrissen. Es folgen aber noch zwei Angaben zur Nordgrenze.

Im Norden sind als Begrenzungen genannt ein Graben („fossa“), vielleicht zu verstehen als ein mit Wasser gefüllter Geländeeinschnitt oder kleiner namenloser Bachlauf, mit dem Namen Hirschsprung („Hirsissprunck“) und ein Hügel oder eine Anhöhe („,collis“), genannt Waidmannshütte („Weydemannissciets“), wörtlich ,des Waidmanns Kiez؛. Eine sichere Lokalisierung der beiden Flurstellen im Gelände ist zumindest bisher nicht möglich. Die naheliegende Vermutung, es könnte sich bei der Anhöhe eine Verbindung mit dem heutigen Ort Weidensdorf nördlich Glauchau und westlich der Mulde, 1161/1171 (Kopie 16. Jh.) „Weidemannesdorff“6, herstellen lassen, ist schon deshalb hinfällig, weil der Ort rund $10 \mathrm{~km}$ nördlich der Mündung des Mülsenbachs an der Mulde liegt und damit nicht zum Parochialgebiet gehören kann.

\section{Das „territorium Zwikowe“ als Neuland für Kirche und Besiedlung}

Es verblüfft natürlich etwas, dass der nördliche Grenzverlauf in der bedeutsamen Urkunde so knapp oder gar dürftig angegeben wurde. Das wird aber verständlich, wenn man den Besiedlungsstand in dem Raum an der Mulde um 1100 näher betrachtet. Da sind im Anschluss an den Altsiedelgau Plisni mit dem 1066 in Schmölln bestehenden Kloster ${ }^{7}$ weiter in süd- östlicher Richtung liegend die Kleinherrschaften Meerane und Mosel westlich bzw. links der Mulde $\mathrm{zu}$ beachten. Beide sind mit großer Wahrscheinlichkeit zum Ausgang des 11. Jahrhunderts bzw. um oder spätestens kurz nach 1100 in der Nachfolge zu den erfolgreichen Bemühungen Wiprechts von Groitzsch als erste deutsche Neugründungen im Landesausbau entstanden. ${ }^{8}$ Damit war zugleich der dünne slawische Siedelstrang an der Mulde erreicht worden. Diese Verbindung mit dem Hause Wiprechts ist durchaus einleuchtend und begründet, wenn man beachtet, dass das Zwickauer Gebiet 1118 als Besitz der Gräfin Bertha von Groitzsch bezeichnet wird. Es liegt nahe, den weiter nach Nordosten anschließenden und schon im Vergleich zur Zwickauer Region etwas früher deutsch besiedelten Landstrich mit den Kleinzentren Meerane und Mosel $^{9}$ als ursprünglich auch zum Einflussgebiet derer von Groitzsch gehörig anzusehen. Auf Zusammenhänge zwischen den frühen kleinen Siedelräumen um Meerane und Mosel hat auch Walter Schlesinger als Landeshistoriker und Kenner des Gebietes von Kindheit an aufmerksam gemacht. ${ }^{10}$ Dazu gehört auch der ausdrückliche Hinweis, dass die Kirchengründung von Zwickau der von Lausick 1105 im Umfeld Wiprechts nachgebildet worden ist, was sich an der Größe der Territorien sowie Anzahl der vorbestimmten Geistlichen erkennen lässt. Nur mit dem Unterschied, dass letztere von Bosau nach Pegau, aber die in Zwickau aus Pegau kamen. ${ }^{11}$

Aus der genauen Grenzbeschreibung ist zugleich klar zu erkennen, dass die Urkunde von 1118 mit der Weihe der Parochialkirche St. Marien erstens weit nach Südosten in das Erz-

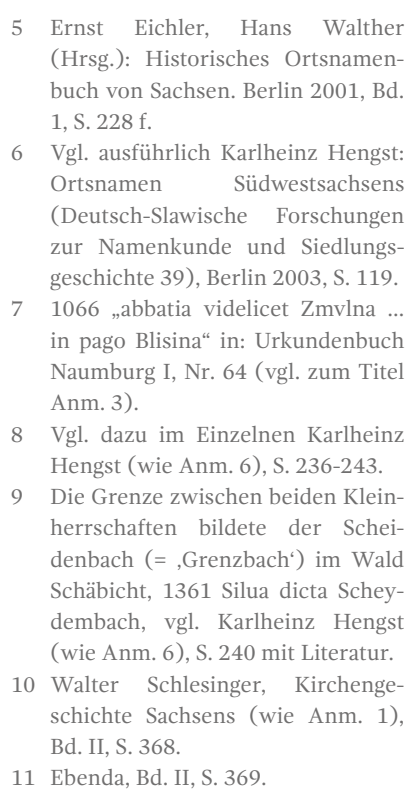

8 Vgl. dazu im Einzelnen Karlheinz Hengst (wie Anm. 6), S. 236-243.

9 Die Grenze zwischen beiden Kleinherrschaften bildete der Scheidenbach (= ,Grenzbach') im Wald Schäbicht, 1361 Silua dicta Scheydembach, vgl. Karlheinz Hengst (wie Anm. 6), S. 240 mit Literatur.

10 Walter Schlesinger, Kirchengeschichte Sachsens (wie Anm. 1), Bd. II, S. 368 .

11 Ebenda, Bd. II, S. 369.

Stadtansicht Zwickaus mit dem Dom St. Marien, Radierung um 1880

๑ SLUB Dresden, Deutsche Fotothek

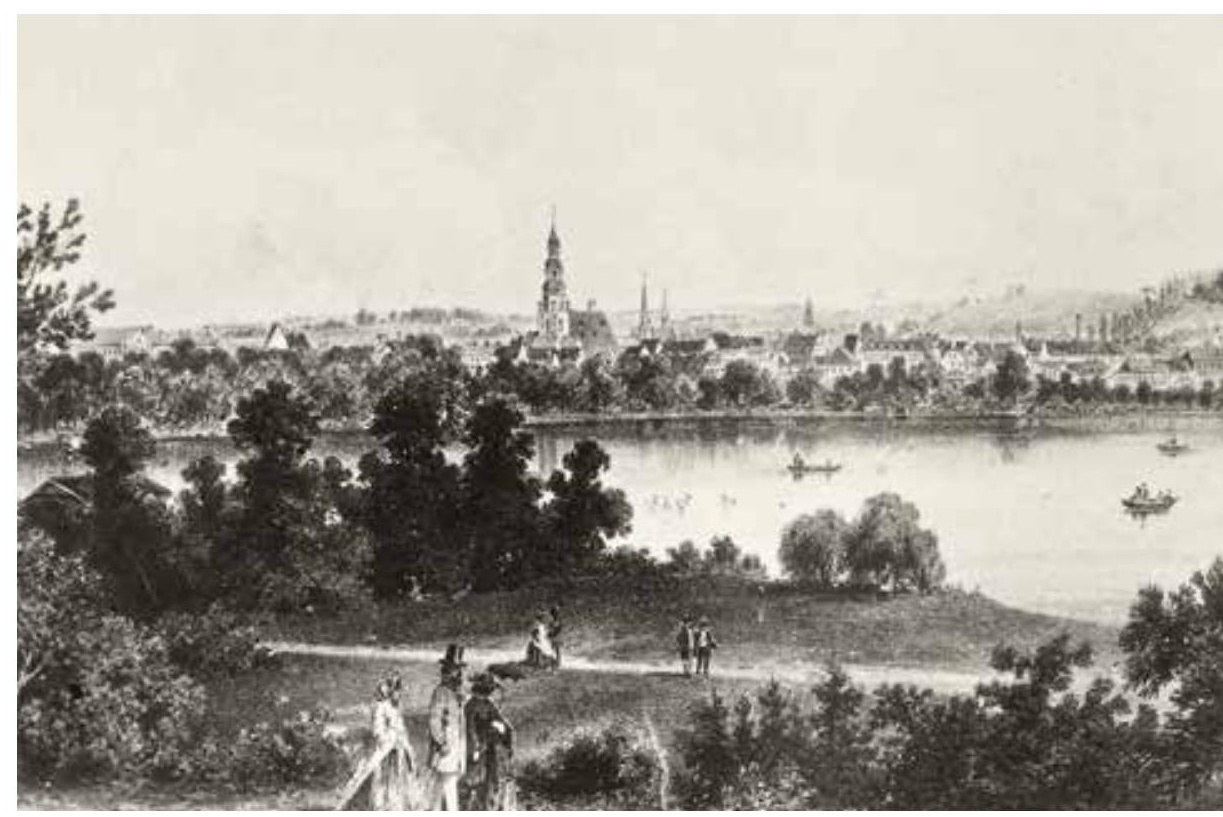


12 Vgl. Ernst Eichler, Hans Walther (Hrsg.): Historisches Ortsnamenbuch von Sachsen. Berlin 2001, Bd. 2, S. 357.

13 Vgl. Walter Schlesinger, Kirchengeschichte (wie Anm. 1), Bd. II S. 367-369.

14 Felix Rosenfeld, Urkundenbuch Naumburg (wie Anm. 3), Nr. 64, S. 57.

15 Vgl. Historisches Ortsnamenbuch von Sachsen (wie Anm. 12), Bd. 2., S $370 \mathrm{f}$. Die von mir abweichend angegebene feminine slaw. Ausgangsform entspricht der Urkundenform und erklärt sich durch gedanklich zu ergänzendes altsorb. „veśc ,Dorf:.

16 Die Angaben folgen verkürzt dem Historischen Ortsnamenbuch von Sachsen (wie Anm. 12). Neuere Erklärungen werden - außer bei Planitz - gesondert angemerkt. Ausführlichere Darstellungen zu den einzelnen Siedlungsnamen finden sich bei Karlheinz Hengst, Ortsnamen Südwestsachsens. Berlin 2003 (wie Anm. 6); vgl. Walter Schlesinger: Kirchengeschichte (wie Anm. 1), Bd. 2, S. 368 mit Verweis auf die vordeutschen, also slawischen Dörfer von der heutigen Wüstung Grabau bis Bockwa. gebirgswaldgebiet hineinreicht, zweitens die Mulde dabei in der Gebietsbestimmung nach Osten überschreitet und im Süden bis zur Mündung des Schwarzwassers in die Mulde führt. Es handelt sich also um ein Gebiet, das später erst zum Bistum Meißen gehörte.

Drittens ist westlich der Mulde der siedlungsfreie Raum um die heutigen Städte Schneeberg und Kirchberg bis Ebersbrunn und zur Pleiße hin bis an die Grenze zur Kleinherrschaft Mosel erfasst. Mit anderen Worten: Das damals siedlungsleere Territorium entlang des Mülsenbachs bis zur heutigen Stadt Aue, von dort nach Westen bis Ebersbrunn und Steinpleis und zurück an die Mulde bei Crossen wurde vom Bischof der Kirche St. Marien als künftig $\mathrm{zu}$ versorgendes Gebiet zugewiesen.

Ausdrücklich vermerkt die Urkunde noch zweierlei. Einmal, dass das umschriebene Gebiet künftighin zu bebauen und zu nutzen ist. Und zum anderen, dass die Bewohner dann gemäß dem Willen von Gräfin Bertha auch den Zehnten aus Fischfang und Jagdertrag zur Versorgung der Geistlichen erbringen sollen. Damit wird einerseits der Auftrag zum Landesausbau mittels Neuansiedlung erteilt, andererseits auch im Hinblick auf die ansässigen Slawen entlang der Mulde von Crossen bis Bockwa die Einbeziehung in die Zehntleistung erteilt. Die im Kloster Bosau bei Zeitz ausgefertigte Urkunde nennt 16 hochrangige Zeugen. Davon sind fünf aus dem geistlichen Stand, nämlich zwei Pröbste („prepositi“) und drei Stiftsherren („canonici“). Unter den elf Edelfreien sind u. a. Graf Heinrich („Henricus comes“) als Bruder der Gräfin Bertha und mit näherer Sitzangabe Werner von Schkeuditz („Wernherus de [S]cudicz“).

\section{Das Gebiet an der Mulde bis 1118}

In jener Zeit war das Gebiet entlang der Mulde noch von den weiten Wäldern des Miriquidi umgeben. Vereinzelte Ansiedlungen gab es südlich der slawischen Altsiedelgaue Rochelinze und Plisni mit seinem Zentrum Altenburg nur entlang der Mulde, also weiter südlich von Schlagwitz, dem Dorf der Leute eines Slavek. ${ }^{12}$ Wie im gesamten Gebiet östlich der Saale bildeten die Slawen auch nach der deutschen Eroberung dieses Raumes um 930 n. Chr. weiterhin die Bevölkerungsmehrheit, wenn auch im oberen Muldengebiet nur schwach vertreten.

Die weltlichen und kirchlichen Obrigkeiten hatten ihre Sitze in den Burgwardzentren der 968 gegründeten Bistümer Merseburg, Zeitz/ Naumburg und Meißen. Von da aus erfolgte seitdem zielgerichtet, aber auch sehr allmählich die Missionierung, also die Christianisierung des slawisch bewohnten Landes, worauf schon Walter Schlesinger vor Jahrzehnten in seiner Kirchengeschichte Sachsens hingewiesen hat. ${ }^{13}$ Ein frühes Zeugnis dafür ist das 1066 erwähnte Kloster in Schmölln, „abbatia [...] Zmvlna [...] in pago Blisina“. ${ }^{14}$ Gegründet wurde es in dem slawischen Ort „Smol'na“, einer Niederlassung zur Harz- und Pechgewinnung. ${ }^{15}$ Das Kloster lag also noch westlich von der Pleiße an der Sprotte im Süden des slawischen Altgaues Plisni.

In jener Zeit der Regentschaft des letzten Saliers, die zwar das Ende des bekannten Investiturstreites unter Kaiser Heinrich IV. brachte, standen die östlichen Gebiete weniger im Blickfeld deutscher Reichspolitik. Das änderte sich aber bei dem Vertrauten von Heinrich IV., dem bekannten Wiprecht von Groitzsch. Er veranlasste in der zweiten Hälfte des 11. Jahrhunderts Landesausbau durch Gründung von deutschen Ansiedlungen an der Weißen Elster im Umfeld von Groitzsch im Südwesten von Leipzig. Dazu kam, dass Wiprecht die Tochter des Herzogs von Böhmen geheiratet hatte und das Bündnis des böhmischen Herrscherhauses mit Heinrich IV. so festigte, dass letzterer den böhmischen Herzog zum König von Böhmen ernannte. Infolgedessen standen auch die wegemäßigen Verbindungen nach Süden über das Erzgebirge sowie das Verringern der Gebiete ohne jegliche Bewohnerschaft entlang einer solchen Trasse („semita Bohemica“) besonders im Blickfeld. Die Geschehnisse von 1118 auf Initiative von Gräfin Bertha sind also in einem größeren Zusammenhang durchaus verständlich und nachvollziehbar.

Durch den Bischof im Bistum Naumburg erfolgte 1118 auf Veranlassung der Gräfin Bertha von Groitzsch eine Erweiterung seines Bistums nach Süden. Die Urkunde zur Weihe der Marien-Kirche nennt keine Orte. Dennoch bestanden damals einige Siedlungen entlang der Mulde. Sie waren von Slawen angelegt worden, die allmählich wohl vor allem im 9. Jahrhundert aus dem Altsiedel-Raum Rochlitz dem Verlauf der Mulde folgend nach Süden hin flussaufwärts siedelten.

Während die Masse der heutigen Orte in dem Pleißen- und Muldenraum nach Süden hin ihre Entstehung dem großen deutschen Landesausbau seit Mitte des 12. Jahrhunderts in der Zeit von Kaiser Friedrich I. Barbarossa verdankt, lassen sich für das 9. und 10. Jahrhundert einige slawische Siedelplätze für das Territorium Zwickau, also den Muldenraum mit dem Gebietsnamen „Zwicowe“, nachweisen. Die deut- 
schen Obrigkeiten haben in ihren Kanzleien die Namen von slawisch benannten Orten und anderen geographischen Objekten, vor allem von Bächen, recht genau nach der damaligen Aussprache und Sprechweise der Slawen festgehalten.

\section{Die slawischen Siedlungen im Parochial- gebiet von St. Marien im Jahr 1118}

Obwohl die Weiheurkunde von 1118 keine Ortschaften oder Siedelplätze innerhalb des in der Grenzbeschreibung umrissenen Gebietes nennt, hat es solche dennoch gegeben. Für den Bischof bestand kein Anlass, diese bei Ausstellung der Urkunde zu erwähnen. Das damals zum Besitz der oben bereits genannten Bertha von Groitzsch gehörige Gebiet wird mit einer Kirche ausgestattet. Deren Einzugsgebiet reicht von da ab bei Schlunzig östlich über die Mulde hinaus bis zum gesamten Mülsengrund und erfasst den Muldenraum zu beiden Seiten von Schlunzig aus weit nach Süden bis zur Schwarzwassermündung in die Mulde bei der heutigen Stadt Aue. Die folgenden Orte an der Mulde flussaufwärts lassen sich auf Grund ihrer sprachlichen Struktur sowie ihrer ursprünglichen Orts- und Flurformen als die ältesten slawischen Ansiedlungen bestimmen. ${ }^{16}$ Es sind Platzdörfer mit Block- und Streifenflur: ${ }^{17}$

- Schlunzig, 1219 „Slunz“, 1413 „Slunczke“, zu altsorbisch „Slǫčsk-“ (Ort an Flusskrümmung);

-Wulm, 1219 „Vulmin duo“, zu altsorbisch „Volmin-“ (Ort, wo Fluss Wellen, Wogen zeigt);

- Crossen, 1219 „Crozne“, wahrscheinlich ein Name ohne Suffigierung mit Hinweis auf ein Handwerk zu altsorbisch „Krosna“ (Webstuhl); ${ }^{18}$

- Pölbitz, 1219 „Belwiz“, zu altsorbisch „Bělovica“ (Ort an feuchter Wiese);

• Osterwein, [1212] „Osterweine“, [1219] „Osterwegen“, 1329 „Osterwen“, zu altsorbisch „Ostrov'ane“ ,Inselbewohner' oder evtl. auch „Ostrov'no“ (Ort auf Insel). ${ }^{19}$ Der Ort ist in Zwickau aufgegangen.

- Schedewitz, 1219 „Schetwiz“, 1322 „Czethewitz“, zu altsorbisch „Četov-c-“ bzw. „Četovic“ etwa (Ort einer bewaffneten Schar); ${ }^{20}$

- Bockwa, 1219 „Bukwen“, zu altsorbisch „Bukov'ane“ (Ort der Leute am Buchenwald).

Weitere Orte, die zwar einen slawischen $\mathrm{Na}$ men weiterführen, beruhen jedoch vor allem auf slawischen Bachnamen, vereinzelt auf einem Namen für Bienenzucht bzw. auf besonderem Tiervorkommen. Es sind dies aber keine slawischen Ansiedlungen gewesen:
• Pöhlau, 1338 „von der Bele“, 1405 „die Behl“, zu altsorbisch „Běla (voda, rěka)“ etwa ,Weißbach';

•Planitz, [1192] „Plaunizc“, 1243 „Plawnicz“, zu altsorbisch „Plav'nica“ (Bach mit Schilfröhricht und überschwemmtem Umland);

- Vielau, [1240 „Bilowe“], zu altsorbisch „Bělava“ (Weißbach) oder „Běl'ava“ (Bach in feuchter Wiese);

- Crinitz, 1388 „Crinicz“, zu altsorbisch „Krinica“ (Bach in schüsselförmiger Vertiefung im Gelände);

- Culitzsch, 1388 „Kulcz, Kultzsch“, zu altsorbisch „Kolč/Kulč“ (hohler Baumstock, Bienenstock);

-Wilkau, 1432 „Wilkaw“, zu altsorbisch „Vil'kov-“ (Ort/Stelle, wo es Wölfe gibt).

Diese letztgenannten sechs Orte führen slawische Flurnamen fort. Sie wurden vor der deutschen Besiedlung von den Slawen geprägt und dienten ihnen zur Orientierung im weiteren Umland. In der Zeit der deutschen bäuerlichen Zusiedlung ab Mitte des 12. Jahrhunderts wurden die Namen als Ortsnamen weiterverwendet. In allen Fällen haben wir es mit den für jene Zeit typischen Waldhufendörfern zu tun.

Im engeren „territorium Zwikowe“ waren somit 1118 existent als kleinere slawische Ansiedlungen Crossen, Pölbitz, Osterwe(i)n, Schedewitz und Bockwa. Die Kirchengründung erfolgte also in einem sehr schwach besiedelten Gebiet. Zugleich ist gemäß der von Nordwesten nach Südosten voranschreitenden Landesausbaurichtung ablesbar, dass die Kirchweihe auch
17 Vgl. die Angaben jeweils unter dem Ortsnamen in: Historisches Ortsverzeichnis von Sachsen. Neuausgabe. Hrsg. von Karlheinz Blaschke, 2 Halbbände, Leipzig 2006.

18 Die zahlreichen vergleichbaren $\mathrm{Na}$ men im westslawischen Sprachraum bereiten noch immer Schwierigkeiten, da die urslawische Basis mit dem zugrunde liegenden Motiv ungeklärt ist. Daher wurde auch versucht, bei dem Lexem neben vorwiegend mit der Bedeutung ,Webbalken' auftretender Semantik auch die Angaben ,Gestell oder ,Korb“ mit dem Fischfang zu verbinden

19 Ausführlicher vgl. Karlheinz Hengst, Formale Quellen zu Sprache und Geschichte im Mittelalter, in: R. Aurig (Hrsg.): Im Dienste der historischen Landeskunde. Beucha 2002, S. 77-93.

20 Ausführlich dazu Karlheinz Hengst: Schedewitz und Zschocken - zwei Wegesicherungen an Böhmischem Steig, in: Onomastica Slavogermanica XXV, Stuttgart/Leipzig 2008 , S. 24-28.

Die St. Marienkirche in

Zwickau vor dem Brand 1650,

Federzeichnung

๑ SLUB Dresden, Deutsche Fotothek

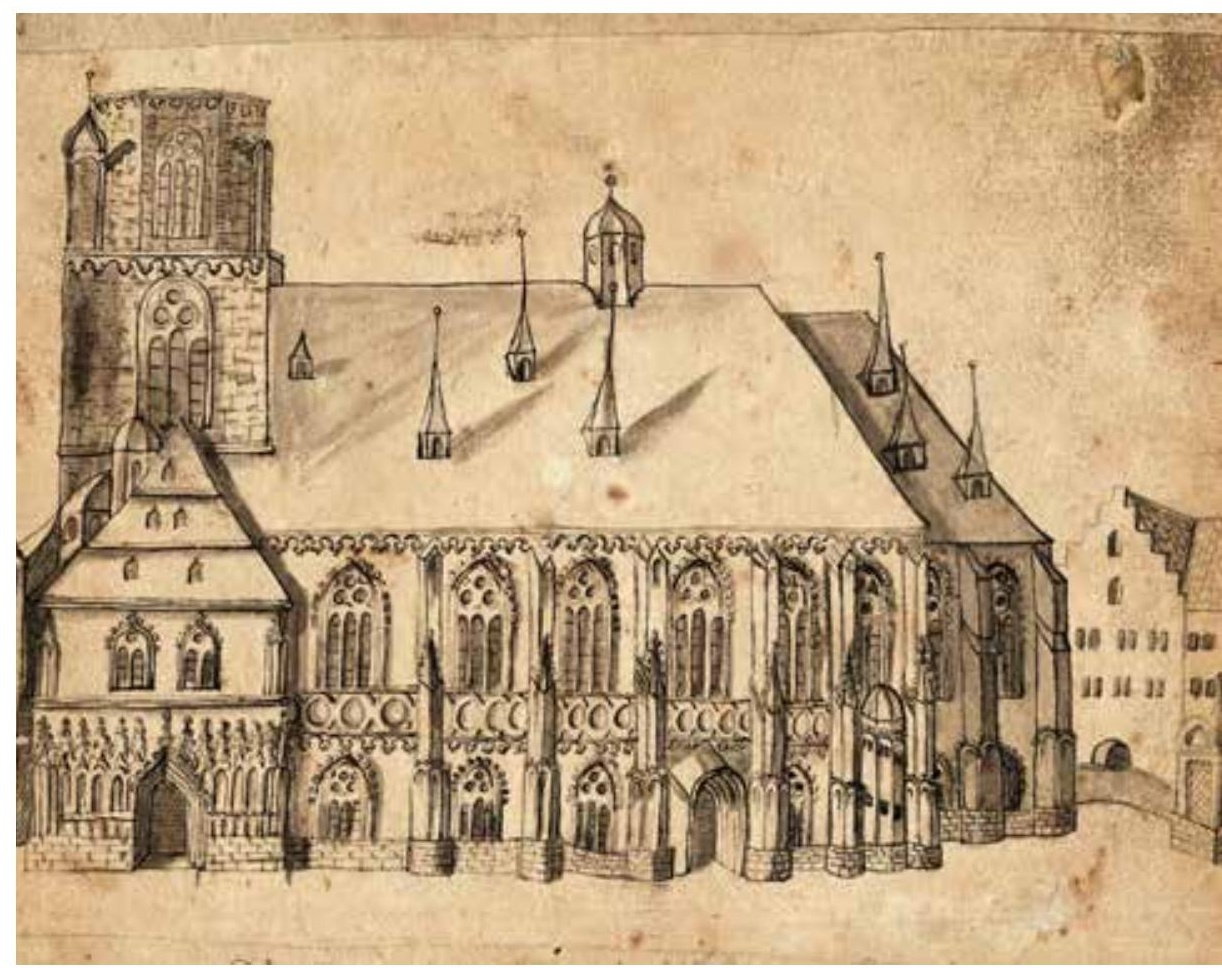


21 Im Urkundenbuch Naumburg (Anm. 3) I, Nr. 116 ausdrücklich angeführt, da die Urkunde von 1118 nur in viel späterer Abschrift erhalten geblieben ist. Die Graphie $<\mathrm{Zcw}>$ dürfte auf Modernisierung durch den Kopisten im 16. Jh. beruhen. Auch auslautend <aw > zeigt die seit Ausgang 14. Jh. übliche Gestalt, während die Urkunden mit diesem Namen im 12. und 13. Jh. $<$ owe $>$ haben. Das <aw > reflektiert also eine spätere Entwicklung zu gesprochenem -au.

22 Vgl. K. Hengst: Zwickau - Geschichte eines Namens, in: Namenkundliche Informationen 69 (1996), S. 74.

23 Ernst Eichler: Slawische Ortsnamen zwischen Saale und Neiße. Bd. 4. Bautzen 2009, S. 148

24 Vgl. Urkundenbuch Naumburg I, Nr. 123, 175, 191, 192, 212, 238, 281.

25 Vgl. Hans Patze: Altenburger Urkundenbuch. Jena 1955, Nr. 60.

26 Vgl. A. Profous: J. Svoboda, Místní jména v Čechách [Ortsnamen in Böhmen]. Bd. 4, Praha 1957, S. 794. 27 Ebenda S. 793. bereits diesem Trend entsprach und zu erkennen gibt, im oberen Muldenraum künftige Rodung und Neuansiedlung zu betreiben. Das enthält die Urkunde von 1118 im abschließenden Teil auch ausdrücklich als Aufgabenstellung.

\section{Die Herkunft des Stadtnamens „Zwickau“}

Das dazu vorgesehene Gebiet war auch in den Kanzleien mit Namen bekannt. Der 1118 erstmals genannte Name für das heute geläufige Zwickau galt damals nicht für einen Ort, sondern für ein Gebiet an der Mulde. In einem Regest nach dem Original der Urkunde ist zu lesen „in territorio Zwickaviensis“. ${ }^{21}$ Diese am ehesten dem Original entsprechende Schreibung weist auf eine Anlautgraphie $\langle\mathrm{zw}\rangle$ hin. Auf Grund der slawischen Siedlungsnamen an der Mulde besonders im Raum von Zwickau ist die Forschung auch bei Zwickau von einem slawischen Namen ausgegangen. ${ }^{22}$ Bei allen Bemühungen ließ sich aber das Motiv für die Namengebung nicht klar ermitteln und nur mit einem vermutlich auffallend hellen Geräusch beschreiben. ${ }^{23}$ Die bisherige slawische Erklärung ist nun nach eingehenden Untersuchungen zu slawischen Gebietsnamen hinfällig geworden. Solche Namen zeigen eine gänzlich andere Struktur und niemals das auslautende toponymische slawische Suffix -ov. Der Gebietsname Zwicowe, später auch Zcwickaw, beruht nach dem heutigen Erkenntnisstand nicht auf slawischer, sondern auf deutscher Namengebung. Es handelt sich dabei um den Namen für ein Landstück zwischen der Mulde, Moritz- und Planitzbach. Dieses Territorium wurde nach seiner Lage zwischen den Wasserläufen mit mittelhochdeutsch zwic (dazu der Genitiv -ckes) ,Keil, Zwischenstück‘ (vgl. deutsch Zwickel) plus mittelhochdeutsch ouwe ,Aue“ gekennzeichnet. Die „Zwick-Aue“ war also der deutsche Name für genau jenen kleinen „Landstreifen“, den die Slawen im 9. Jahrhundert als Insel empfunden hatten und daher für eine Siedlung dort den Namen *Ostrov'ane ,die Inselbewohner" oder evtl. auch *Ostrov'no ,Inselort“ geprägt hatten (vgl. oben). Die lange erhalten gebliebene Mundartform de zwicke für Zwickau bestätigt die deutsche Bildung mit dem femininen „die“ infolge von "die Aue“.

Es ist anzunehmen, dass der Name spätestens seit dem 10. Jahrhundert gebraucht wurde. Er ist von der deutschen Obrigkeit übernommen und beibehalten worden, was die Urkunde zur Kirchenweihe von 1118 ausweist.

Alle nach 1118 in Urkunden vorkommenden Schreibungen zeigen im 12. Jahrhundert nur die Anlautschreibung <zw>: 1121 „in Zwicowe“, 1145 „in Zwicowe“, 1151 „in Zwickowe“, „in pago Zwickowe“, 1152 „in Zwikowe“, 1160 „in Zwikowe“, 1171 „in pago Zwikowe“, ${ }^{24}$ auch 1206 „Zvvickovve“. ${ }^{25}$ So wie der Anlaut in der Schrift variieren konnte, treten auch die Schreibungen mit $<\mathrm{c}>$ oder $<\mathrm{k}>$ sowie $<\mathrm{ck}>$ als gleichwertig für gesprochenes $/ \mathrm{k} /$ in der Mitte des Namens auf. Der sicher bald nach 1118 als Ortsname gebräuchlich gewordene frühere Gebietsname hat im Auslaut mittelhochdeutsch „ouwe“ (Aue) gutbewahrt. Für die gesamte Namensgebung der Slawen im Gebiet an der Mulde südlich vom Altsiedelraum Rochlitz ist eines auffällig: Alle Namen sind nach Erscheinungen aus der Natur bzw. nach geographischen Gegebenheiten, nur vereinzelt auch nach Tätigkeiten, gegeben worden. Die Umwelt hat die entscheidenden Motive für die Namenbildung geliefert. Es erfolgte keine Vergabe von Namen nach Personen, die sich mit ihrer Familie niederließen, wie dies vorher in der Zeit der Landnahme in den Altsiedelräumen geschah.

\section{Die Urkunde von 1118 in ihrer Bedeu- tung für die Besiedlungsgeschichte}

Die sprachgeschichtliche Betrachtung und Erklärung der slawischen Ortsnamen im engeren Zwickauer Raum bringt auch in Verbindung mit der Urkunde von 1118 neue Erkenntnisse insbesondere zur Besiedlungsgeschichte, was hier nur kurz mitgeteilt werden kann. 1118 und auch später wird der Böhmische Zoll in beachtlicher Höhe erwähnt. Das hat immer wieder zu der Frage geführt, wo sich die Zollstätte befunden haben könnte. Bisher wurde sie im engeren Gebiet der späteren mittelalterlichen Stadt Zwickau gesucht. Inzwischen hat sich jedoch Folgendes gezeigt: Der ein ganzes Stück entlang der Mulde verlaufende alte Böhmische Steig und sein vermutlich älterer Verlauf durch den Mülsengrund hat sehr wahrscheinlich am unteren Ortsende des heutigen Zschocken diese Zollstelle besessen. 1219 „Schakan“, [1286] „Scacken“, 1358 „czu dem Czschackan“ usw. führt zu altsorbisch „Čakan“, einer Bildung $\mathrm{zu}$ einem Personennamen „Čakan“ (der Erwartete) + -j-Suffix oder zu „Čakany“ (die [Leute mit] Streitäxten) zu „čakan“ (Streitaxt), wobei die Slawen Ende des 10. Jahrhunderts die den Zoll erhebenden bewaffneten Wachleute nach ihrer Bewaffnung benannt haben könnten. ${ }^{28}$ Archäologisch ist am Südwestende des Ortes ein Bühl mit dem Namen „Waal“ nachgewiesen. Das ist mit hoher Wahrscheinlichkeit die Zollstätte gewesen. 
Sie lag innerhalb der in der Urkunde 1118 beschriebenen Grenzen, und sie bestand bereits im 11. Jahrhundert - fraglich, ob gar schon im 10. Jahrhundert. Die Nähe der erst später angelegten Burgen Hartenstein und Wildenfels dürfte auch nicht ganz zufällig sein. In Schedewitz befanden sich wahrscheinlich spätestens seit der Mitte des 10. Jahrhunderts die „stationierten“ Bewaffneten. Die Slawen haben also beide Namen geprägt. Über die ethnische Zugehörigkeit der Waffenträger ganz im Süden des noch schwach besiedelten Muldenraumes erfahren wir nichts. ${ }^{29}$

Vom Raum Rochlitz aus gesehen waren Schedewitz und auch Zschocken wichtige Standorte mit gewissen Verwaltungsaufgaben in den Jahrzehnten nach 930, also seit der Eingliederung des Ostsaaleraumes ins Reich. Es ist dabei auch nicht ausgeschlossen, dass die aus dem Chemnitzer Raum bekannten Wolfsjäger möglicherweise auch in Schedewitz einen Stützpunkt hatten. Auf ein Betätigungsfeld der Wolfsjäger weisen zwei Namen recht deutlich hin. Das ist einmal der oben angeführte Name altsorbisch „Vil'kov-“ (Wolfsort). ${ }^{30}$ Und zum anderen ist es die Angabe eines Grenzpunktes in der Urkunde von 1118 mit dem Namen „mons Luderni“ (vgl. oben). Besonders im Winter ließ sich das Raubwild im frischen Schnee vom Luderplatz aus gut aufspüren. ${ }^{31}$

Zeichen für die deutsche Namengebung infolge von der Obrigkeit schon früh veranlasster weidmännischer Tätigkeit bietet die Urkunde auch noch mit zwei Angaben zu den nördlichen Grenzpunkten: ein Graben (Bach) „Hirsissprunck“ und ein Hügel „Weydemannissciets“, also ,Hütte eines Jägers“ (vgl. oben). Und als ein weiterer Stützpunkt ist der heutige Ort Weidensdorf bei Glauchau recht wahrscheinlich, im 12. Jahrhundert „Weidemannesdorff" (vgl. oben).

\section{Die Zuverlässigkeit der Urkunde von 1118}

Insgesamt ist die Urkunde von 1118 in ihrer Abschrift aus dem 16. Jahrhundert als sehr zuverlässig zu bewerten. Das unterstreichen auch die für weitere Grenzpunkte angegebenen Namenformen „fons Albodistudinza“ für die Pleißenquelle mit einem deutschen Personennamen „Albold“ und die altsorbischen Formen „Studenica“ zu „studen-“ (kalt) sowie „Scurnice“ für „Čornica“, das heutige gleichbedeutende ,Schwarzwasser“. „Albodistudinza“ ist eine slawische Bildung für (Ort des Albold an kalter Quelle $)^{32}$ mit einem ersten deutschen Personennamen im Zwickauer Raum. Der Name von
1118 ist die Niederschrift einer slawischen Form nach Gehör noch aus dem 10./11. Jahrhundert. Hier dürfte es sich um eine Versorgungsstelle gehandelt haben. Diese haben die Slawen benannt nach einem von Rochlitz her eingesetzten Beauftragten mit Siedelhof. Die Sicherung der Verbindungswege wurde also offensichtlich von der deutschen Obrigkeit zielstrebig betrieben. Es ist daher auch nicht zufällig, dass zuerst an dem am weitesten westlich gelegenen Weg von Altenburg bzw. Rochlitz aus gen Süden diese Wegesicherung mit Einrichtung einer Zollstelle erfolgte und dann auch da die erste Kirchengründung in Angriff genommen wurde.

Die Namenformen „Scurnice“ und „Slunczke“ sind sprachwissenschaftlich aufschlussreich. ${ }^{33}$ Sie bezeugen in ihrer lautlichen Struktur, dass die Namen im Muldenraum spätestens um die Mitte des 10. Jahrhunderts in Registraturen der markgräflichen und bischöflichen Kanzleien erfasst und im Verwaltungsbereich von da an weiter verwendet wurden. Besonders beachtenswert für das Miteinander von Slawen und Deutschen im Muldenraum vom 10. bis 12. Jahrhundert ist fernerhin die Übertragung der Namen von slawisch benannten Gewässern auf die nach 1150 an diesen angelegten Dörfer.

\section{Das Umfeld und der Bestand der Parochie „Zwikowe“ um 1118}

Die zu den Herrschaften Meerane bzw. Mosel gehörigen Dörfer gehörten zum Weiheakt nicht in den Parochialbereich. Bei Meerane waren damals Zubehör Seiferitz, Dennheritz, Schindmaas, Lipprandis, Dietrich ${ }^{34}$ und bei Mosel die Dörfer Oberrothenbach, Helmsdorf, Weißenborn und Niederhohndorf ${ }^{35}$ sowie das Anfang des 15. Jahrhunderts noch bestehende Rappendorf $^{36}$. Damit wird auch verständlich, dass westlich der Mulde erst ab dem Dorf Marienthal das Zugehörigkeitsgebiet der frühen Parochie von St. Marien beginnt. Eine Karte zeigt die Urpfarrei mit ihrem Zuwachs im Laufe der Jahrhunderte. ${ }^{37}$

Als erste Neugründung nach 1118 darf demnach Marienthal gelten. Es wurde nach der Kirchenheiligen benannt: 1192 (Kopie 14. Jh.) „villa que vallis sancte Marie nuncupatur“ (Dorf, das Thal der heiligen Maria genannt wird). ${ }^{38}$ Die erst relativ spät einsetzende urkundliche Überlieferung darf aber nicht darüber hinwegtäuschen, dass das Dorf sicher bereits bald nach der Weihe der Kirche St. Marien entstanden ist, wahrscheinlich also bereits in der ersten Hälfte des 12. Jahrhunderts. Daran
28 Dazu vgl. in allen Einzelheiten K. Hengst: Schwierige Ortsnamen Westsachsens, in: Onom. Slavogerm. (wie Anm. 21), S. 28-39.

29 Vol. auch die sehr ausführlichen Erläuterungen bei K. Hengst: Namen von Kirchen und Dörfern im Zwickauer Raum als Geschichtsquellen bis zum Ende des 11. Jahrhunderts, in: Zur Kirche gehört mehr als ein Kruzifix. Hrsg. von Michael Beyer et al., Leipzig 2008, S. 25-41.

30 Die sprachlich mögliche Zuordnung zu einem slawischen Personennamen scheidet insofern aus, als es sich um eine Gründung der Siedlung nach Mitte des 12. Jahrhunderts handelt. In dieser Zeit sind neue Siedlungen mit Bezus auf einen slawischen Lokator als Mischnamen gebildet worden mit slawischem Personennamen + Grundwort -dorf.

31 Vgl. Irmgard Lorenz: Russische Jagdterminologie. Analyse des Sprachgebrauchs der Jäger, München 1978, S. 238.

321303 „Alvolsburn“ setzt den Personennamen mit Grundwort -born und später -brunn in der Zeit der mehrheitlich deutschen Besiedlung fort bis zum heutigen „Ebersbrunn".

33 Vgl. dazu ausführlicher und näher erläuternd Karlheinz Hengst: Ortsnamen Südwestsachsens (Deutsch-Slawische Forschungen zur Namenkunde und Siedlungsgeschichte 39), Berlin 2003, S. $102 \mathrm{f}$ und 155.

34 Vgl. Walter Schlesinger in Historische Stätten Deutschlands, Bd. Sachsen. Stuttgart 1965, S. 222 f.

35 Vgl. Walter Schlesinger, ebenda, S. 236.

36 Rappendorf nordwestlich von Zwickau am Oberlauf des Weißenborner Baches ist belegt 1419 „in steynplissen, in villa Rappendorff (StHA Dresden Copial 34, Bl. 49b.) Zugrunde liegt der deutsche Kurzname „Rappo“ zum Personenname „Radboto“ - im 12. und 13. Jahrhundert mehrfach im Raum Plisni belegt. Möglicherweise handelt es sich um den Namen eines Herrn von Mosel, die im 13. sowie 14. Jahrhundert in den Personennamen „Reinboto“ (Anlaut R- und das Zweitglied -boto) zeigen.

37 www.kirche-zwickau.de (29.09.2016). 
38 Urkundenbuch Naumburg I (wie Anm. 3), Nr. 377.

39 Urkundenbuch Naumburg I (wie Anm. 2), Nr. 124, S. 107-110.

40 Vgl. Walter Schlesinger, Kirchengeschichte (wie Anm. 1), Bd. II, S. 192-193.

41 Codex diplomaticus Saxoniae regiae II 6, Nr. 302. Zum Fernhandelsmarkt vgl. Walter Schlesinger, Kirchengeschichte (wie Anm. 1), Bd. II, S. 25.

42 Vgl. Walter Schlesinger: Kirchengeschichte (wie Anm. 1), Bd. II, S. 194-195.

43 Vgl. Urkundenbuch Naumburg (wie Anm. 2) I, Nr. 277, S. 260 f.

44 Vgl. Walter Schlesinger: Kirchengeschichte (wie Anm. 1), Bd. II, S. 194.

45 Codex diplomaticus Saxoniae regiae I 2, Nr. 308, S. $210 \mathrm{f}$.

46 Codex diplomaticus Saxoniae regiae I 2, Nr. 397. S. $275 \mathrm{f}$

47 Vgl. Walter Schlesinger: Kirchengeschichte Sachsens im Mittelalter. Bd. II. Köln, Graz 1962, S. 55.

8 Ebenda S. 5.

49 Ebenda S. 5-6.

50 Vgl. zum Landesausbau ausführlicher Rudolf Kötzschke, Hellmut Kretzschmar: Sächsische Geschichte. Lizenzausgabe Augsburg 1995, S. 88-96; Karl Czok (Hrsg.): Geschichte Sachsens. Weimar 1989, S. 105-123; Karlheinz Blaschke: Geschichte Sachsens im Mittelalter, Berlin 1990, S. 77-110.

51 Walter Schlesinger, Kirchengeschichte (wie Anm. 1), Bd. II, S. 193.

Prof. Dr. Karlheinz Hengst Chemnitz schlossen sich dann nach und nach die Gründungen von Planitz, Cainsdorf, Rottmannsdorf, Culitzsch, Crinitz bis Kirchberg an sowie östlich der Mulde die Orte im Mülsengrund sowie Schneppendorf, Auerbach, Eckersbach bis Wilkau und Haßlau, Friedrichsgrün und Vielau. Dieser Besiedlungsgang im Zuge des forcierten Landesausbaus vor allem in der Zeit von Kaiser Friedrich I. Barbarossa vollzog sich im weiteren Verlaufe des 12. Jahrhunderts bis zum Anfang des 13. Jahrhunderts. Die Ortsnamenlandschaft einer geographischen Karte war damit zu jener Zeit im Wesentlichen geprägt und hat im Laufe der folgenden Jahrhunderte nur wenige Ergänzungen infolge von Neugründungen durch den Bergbau und die industrielle Entwicklung erfahren.

\section{Die besondere Bedeutung der Weihe von St. Marien 1118 für Sachsen}

Noch zu wenig bekannt ist die gleichsam als Pioniertat zu bezeichnende Weihe der Kirche St. Marien von 1118. Die Einrichtung dieser Urpfarrei in einem nur schwach von Slawen besiedelten Gebiet an der Mulde darf durchaus als der Auftakt zum großen Siedelwerk des 12 . Jahrhunderts in Sachsen gelten, insbesondere für den gesamten Vorerzgebirgs- sowie Erzgebirgsraum. Als weitere „Paukenschläge“ im Sinne von Initiativen zum in der Vergangenheit als „Ostsiedlung“ bezeichneten siedlungsmäßigen Landesausbau folgten nach 1118 in recht dichter Abfolge von den deutschen Herrschern und den Bistümern veranlasst deutliche Impulse. Sie seien hier nur überblicksmäßig genannt:

1122 beurkundet Bischof Dietrich von Naumburg die Gründung der Pfarrkirche zu Plauen mit Pfarrecht im gesamten Dobnagau. ${ }^{39}$

1136 etwa erfolgte die Gründung des Benediktinerklosters Chemnitz durch Kaiser Lothar III. ${ }^{40}$ 1143 bestätigte König Konrad III. die Gründung des Klosters in „Kameniz“ und verfügte die Einrichtung eines Fernhandelsmarktes („forum publicum“).41

1143 nahm König Konrad III. eine Schenkung von 100 Hufen im königlichen Waldland beiderseits der Mulde an das nahe Jena gelegen Kloster Bürgel vor, worauf von dort die Anlage eines Benediktinerinnenklosters in dem slawischen Dorf Remse an der Mulde (nahe Waldenburg) zwischen den Orten Kertzsch und Jerisau umgehend erfolgte. ${ }^{42}$ Bereits 1165/1170 überließ Bischof Udo II. von Naumburg dem Nonnenkloster St. Marien und St. Nicolai an der Mulde in Remse im Tausch das Dorf Weidensdorf nördlich Jerisau. ${ }^{43}$ Auf die Stiftung von Kloster Remse als zeitliche und sachliche Parallele zur Gründung von Kloster Chemnitzer hat vor Jahrzehnten bereits Walter Schlesinger hingewiesen. ${ }^{44}$

1162 bestätigte Kaiser Friedrich I. Barbarossa die von Markgraf Otto von Meißen vorgenommene Gründung des Benediktiner-Klosters Altzelle bei Meißen, die vorher Markgraf Otto von Meißen vorgenommen hatte. ${ }^{45}$

1173 richteten Markgraf Otto von Meißen und Dudo von Meineweh sowie Meinher von Werben, der spätere Burggraf von Meißen, das Augustiner-Kloster nahe der späteren Stadt Aue (bekannt als „Klösterlein Zelle“) ein, was durch Friedrich I. ausdrückliche Bestätigung erfuhr. ${ }^{46}$ Als erste Zeugen sind ausgewiesen der Erzbischof von Magdeburg und Bischof Udo von Naumburg.

Seit den Forschungen von Walter Schlesinger ist das besondere Engagement der Naumburger Bischöfe als Verfechter des Landesausbaus allgemein bekannt. ${ }^{47}$ Und als weltlicher Initiator gilt Lothar III., der als „der erste deutsche König [...] an das Werk Ottos des Großen anknüpfte und der deutschen Ostsiedlung des 12. Jahrhunderts die Bahn wies." 48 Sein Nachfolger, der Staufer Konrad III. hat durchaus mit Tatkraft die Politik seines Vorgängers fortgesetzt. Er begann, das Reichsgut neu $\mathrm{zu}$ organisieren, indem es Burggrafen unterstellt wurde. So traten $\mathrm{zu}$ den bisherigen Burggrafen zu Meißen und Dohna nun solche neu hinzu in Altenburg, Leisnig, Döben, Bautzen und Cottbus. Außerdem band er das Egerland ans Reich. ${ }^{49}$

Die große Welle des Landesausbaus ${ }^{50}$ vollzog sich aber erst unter Friedrich I. in der zweiten Hälfte des 12. Jahrhunderts. ${ }^{51}$ Die von Lothar und Konrad sowie von den Bischöfen von Naumburg eingeleitete Entwicklung setzten nun die Reichsministerialen fort. Im hier besprochenen Gebiet waren das weiter westlich im heutigen sächsischen und thüringischen Vogtland vor allem die Vögte von Plauen, Gera, Weida und Greiz; im Muldengebiet die Herren von Waldenburg, die Schönburger, die Meinheringer zu Hartenstein, die Erkenbertinger zu Stollberg und im Anschluss an den Besitz von Kloster Chemnitz die Herren von Erdmannsdorf sowie die von Schellenberg. Die zuletzt genannten Klostergründungen in den Flussräumen von Mulde und Chemnitz lagen aber bestimmt vor dem von ihnen eingeleiteten und nachfolgenden weiteren Landesausbau. Für das Erzgebirge ist somit der Besiedlungsvorgang für die beiden letzten Jahrzehnte des 12. Jahrhunderts und die erste Hälfte des 13. Jahrhunderts anzusetzen. 